\title{
Short communication: Genomic selection using a multi-breed, across-country reference population
}

\author{
J. E. Pryce, ${ }^{\star 1}$ B. Gredler,† S. Bolormaa, ${ }^{\star}$ P. J. Bowman, ${ }^{\star}$ C. Egger-Danner,‡ C. Fuerst,‡ R. Emmerling,§ \\ J. Sölkner,† M. E. Goddard, ${ }^{\star} \#$ and B. J. Hayes* \\ ${ }^{*}$ Biosciences Research Division, Department of Primary Industries Victoria, 1 Park Drive, Bundoora 3083, Australia \\ †Department of Sustainable Agricultural Systems, University of Natural Resources and Applied Life Sciences Vienna, Gregor Mendel Str. 33, \\ 1180 Vienna, Austria \\ $\ddagger Z u c h t D a t a$ EDV-Dienstleistungen GmbH, Dresdner Straße 89/19, 1200 Vienna, Austria \\ §Institute of Animal Breeding, Bavarian State Research Center for Agriculture, Prof. Duerrwaechter Platz 1, 85586 Poing-Grub, Germany \\ \#Faculty of Land and Food Resources, University of Melbourne, Parkville 3010, Australia
}

\section{ABSTRACT}

Three breeds (Fleckvieh, Holstein, and Jersey) were included in a reference population, separately and together, to assess the accuracy of prediction of genomic breeding values in single-breed validation populations. The accuracy of genomic selection was defined as the correlation between estimated breeding values, calculated using phenotypic data, and genomic breeding values. The Holstein and Jersey populations were from Australia, whereas the Fleckvieh population (dualpurpose Simmental) was from Austria and Germany. Both a BLUP with a multi-breed genomic relationship matrix (GBLUP) and a Bayesian method (BayesA) were used to derive the prediction equations. The hypothesis tested was that having a multi-breed reference population increased the accuracy of genomic selection. Minimal advantage existed of either GBLUP or BayesA multi-breed genomic evaluations over single-breed evaluations. However, when the goal was to predict genomic breeding values for a breed with no individuals in the reference population, using 2 other breeds in the reference was generally better than only 1 breed.

Key words: genomic selection, Holstein, Jersey, Fleckvieh

\section{Short Communication}

Genomic selection refers to selection decisions that are based on genomic EBV (GEBV) (Meuwissen et al., 2001). Genomic breeding values are calculated by first estimating SNP effects in a reference population that has both phenotypes and genotypes. The SNP effects are then multiplied by the genotypes of a separate population (generally younger sires) and summed to form a GEBV. The accuracy of GEBV in selection can-

Received August 12, 2010.

Accepted January 25, 2011.

${ }^{1}$ Corresponding author: jennie.pryce@dpi.vic.gov.au didates depends on the size of the reference population where the prediction equation is derived (Hayes et al., 2009; VanRaden et al., 2009). Assembling large enough reference populations for accurate GEBV prediction is a major challenge, especially for breeds without large numbers of bull progeny tested each year. A key question, therefore, is whether using a multi-breed reference population can be used to increase the accuracy of GEBV in such breeds. Throughout this paper, the accuracy of genomic selection refers to the correlation between EBV, calculated using phenotypic data, and GEBV.

Comparing results from 2 breeds in a reference population to 1 breed in the reference population, Hayes et al. (2009) demonstrated that for some traits, a small increase in accuracy can be achieved. However, they only found an advantage for one method (Bayesian method; BayesA) and not the other method tested (BLUP with a multi-breed genomic relationship matrix; GBLUP). One of the limitations with the study of Hayes et al. (2009) was that the reference population consisted of only 781 Holsteins and 400 Jerseys. Adding other breeds to the reference population could increase the accuracy of GEBV, by allowing selection of SNP that are in high linkage disequilibrium (LD) with QTL because the SNP-QTL association persists across the breeds. Although the SNP-QTL associations cannot be quantified per se, an improvement in the accuracy of prediction could be an indication of this. In this study, the reference population was extended to 3 breeds (Holstein, Jersey, and Fleckvieh), to test the hypothesis that having more than 2 breeds in a reference population improves the prediction of GEBV. The hypothesis that predicting GEBV for a breed not in the reference population using a multi-breed reference (i.e., of 2 other breeds) is better than using a single-breed, was also tested.

Five populations, consisting of a total of 3,101 progeny tested sires, were used in this study: 1) a Holstein 
reference population consisting of 755 Holstein sires; 2) a Holstein validation population of 386 Holstein sires that were younger than the ones from the Holstein discovery population; 3) a Jersey population of 364 sires used in either the reference or validation populations; 4) a Fleckvieh (dual-purpose Simmental) reference population of 1,247 sires; and 5) a Fleckvieh validation population of 349 sires. The Holstein and Jersey populations were from Australia, whereas the Fleckvieh population was from Austria and Germany.

Markers were genotyped using the Illumina BovineSNP50 BeadChip (Illumina, San Diego, CA; Matukumalli et al., 2009). The SNP were ordered by chromosome position using Bovine Genome Build 4.0 (http:// www.ncbi.nlm.nih.gov/projects/genome/guide/cow/). Full details of the SNP editing criteria are reported by Hayes et al. (2009). After applying the editing criteria, the number of SNP available for analysis in each data set was 36,986. Each SNP was biallelic.

To investigate the extent of LD between syntenic markers on the same chromosome in Holsteins, Jerseys, and Fleckvieh, the square of the correlation coefficient, representing the presence or absence of particular alleles at 2 loci $\left(r^{2}\right)$, was calculated using the method of Hill and Robertson (1968).

The traits considered were milk, fat, and protein yields. Daughter yield deviations (DYD) were used and were calculated as sire solutions from the following model: $\mathbf{y}=\mathbf{X b}+\mathbf{e}$, where $\mathbf{y}$ is the vector of observations, $\mathbf{X}$ is the incidence matrix of fixed effects (such as contemporary group, herd-year-season, among others), $\mathbf{b}$ is the vector of sire effects (DYD), and $\mathbf{e}$ is the vector of residuals. The fixed effects models differed, but are the same fixed effect models as used in routine genetic evaluations in each respective country. The mean and scale of DYD was only relevant within each country and in the case of Australia, Jersey and Holstein genetic evaluations are routinely performed separately. Therefore, the DYD were not directly comparable. To remove scale and mean effects, the DYD were standardized to have a mean of 0 and a standard deviation of 1 [i.e., $\mathrm{N}(0,1)$ within breed].

Two methods were used to estimate GEBV, namely GBLUP (Hayes and Goddard, 2008) and BayesA (Meuwissen et al., 2001). The model used for the GBLUP analysis was $\mathbf{y}=\mathbf{1}_{\mathbf{n}} \alpha+\mathbf{Z g}+\mathbf{e}$, where $\mathbf{y}$ is the vector of DYD, adjusted for fixed effects (such as herd-year-season) and breed (Holstein, Fleckvieh, and Jersey); $\mu$ is the mean; $\mathbf{1}_{\mathrm{n}}$ is a vector of ones; $\mathbf{Z}$ is a design matrix allocating records to breeding values; $\mathbf{g}$ is a vector of breeding values; and $\mathbf{e}$ is a vector of random normal deviates. The software ASReml (VSN International,
Hemel Hempstead, UK; Gilmour et al., 2006) was used for the analysis.

The BayesA method (Meuwissen et al., 2001) used in this study was modified to include a polygenic effect (Hayes et al., 2009). The model used was $\mathbf{y}=\mathbf{1}_{\mathbf{n}}^{\prime}+\mathbf{X u}+\mathbf{Z v}+\mathbf{e}$, where $\mathbf{y}$ is the vector of $n$ DYD, corrected for herd-year-season and breed effects for each trait; $\mathbf{1}_{\mathbf{n}}^{\prime}$ is a transposed vector of $1 \mathrm{~s}$; and $\mathbf{X}$ is a design matrix [of dimension number of sires $(n)$ by number of SNP $(m)$; i.e., $n \times m]$, allocating records to SNP effects. Marker effects of 0,1 , and 2 that form $X_{i j}$ are the genotype of animal ${ }_{i}$ at $\mathrm{SNP}_{j}$ and are 11, 12, and 22 , respectively. Vector $\mathbf{u}$ is a $(m \times 1)$ vector of SNP effects, $\mathbf{e}$ is a vector of random deviates, and $\mathbf{v}$ is a vector of polygenic breeding values. The GEBV were calculated as $\mathbf{G E B V}=\hat{\mathbf{v}}+\mathbf{X} \hat{\mathbf{u}}$, where $\hat{\mathbf{v}}$ is the estimated polygenic breeding value and $\hat{\mathbf{u}}$ is a vector of estimated SNP effects.

The accuracy of GEBV was calculated as the correlation between GEBV and EBV [r(GEBV,EBV)] within each validation population (Holstein, Jersey, or Fleckvieh). Current estimated breeding values were obtained from the Australian Dairy Herd Improvement Scheme (ADHIS; Melbourne, Victoria, Australia) and ZuchtData (Vienna, Austria), although they were estimated as part of a joint Austria/Germany evaluation by LFL Grub (Bavaria, Germany). The limitation of using EBV is that the relationship between EBV and true breeding values (TBV) is generally less than 1 , especially for younger bulls that generally comprise validation data sets. In Australia, the correlation between EBV and TBV, estimated by ADHIS, for milk, fat, and protein yield was $0.95,0.93$, and 0.95 for Holstein reference, Holstein validation, and Jerseys, respectively. In Austria and Germany, the r(TBV,EBV) for milk, fat, and protein yield was 0.94 and 0.92 for Fleckvieh reference and Fleckvieh validation bulls, respectively.

To investigate the genomic relationships between the breeds, a principal component analysis of the genomic relationship matrix was performed (McVean, 2009; Figure 1). The first principal coordinate on the horizontal axis of Figure 1 clearly divides the Fleckvieh and Holstein populations. For the first principal coordinate, some overlap exists between Holsteins and Jerseys. The second principal coordinate separates Jerseys from both Holsteins and Fleckvieh. The Fleckvieh, Jersey, and Holstein animals used in this experiment did not share recent ancestors (i.e., in their pedigrees traced back to founder animals). However, it is possible that the Jersey and Holstein animals share some common ancestors before pedigree recording because of crossbreeding 


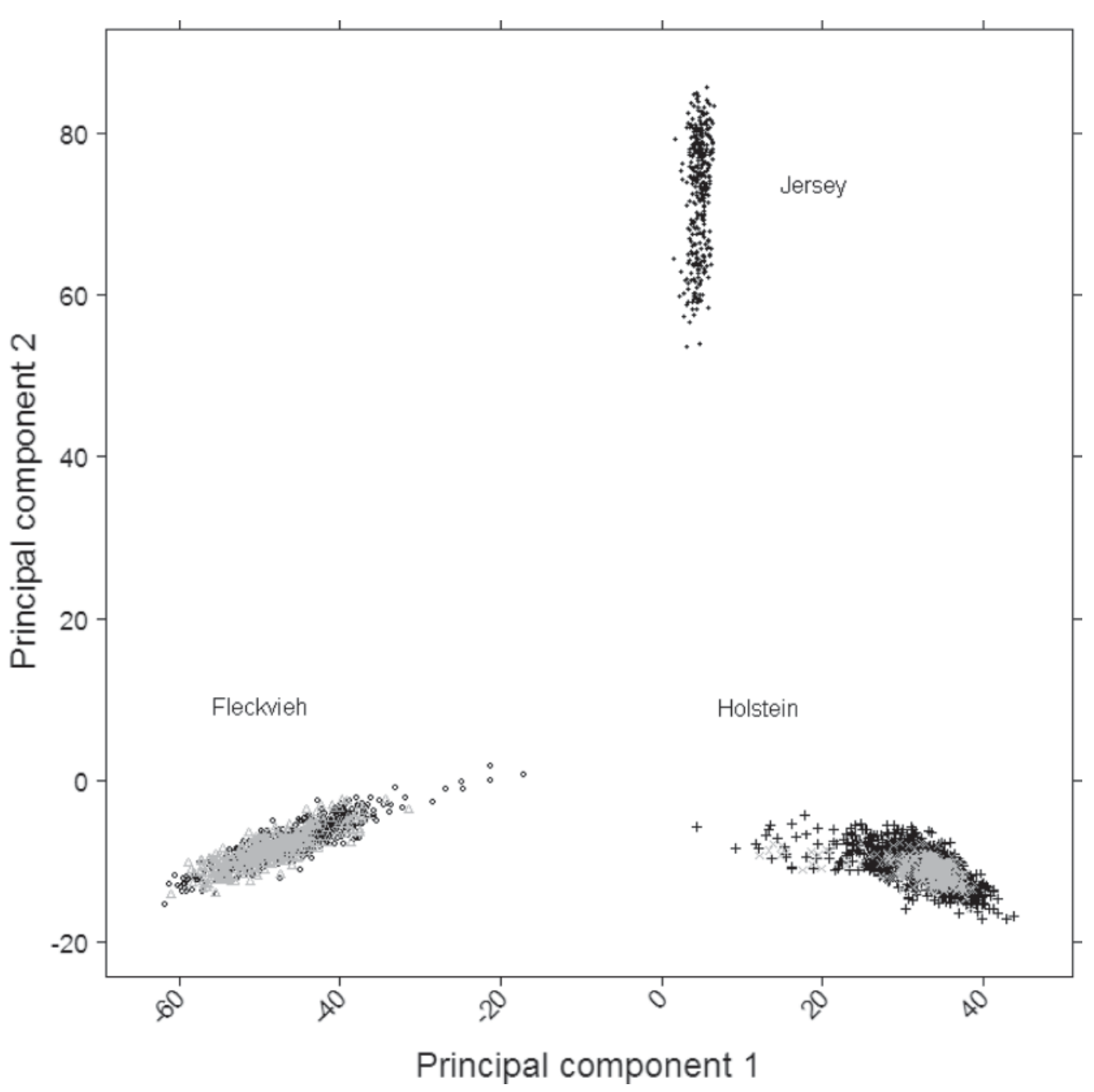

Figure 1. The first 2 principal components (principal components 1 and 2) of Euclidian distances of 5 dairy populations belonging to 3 breeds (Fleckvieh, Holstein, and Jersey); the Fleckvieh and Holstein reference populations are depicted in black, whereas their validation populations are depicted in gray.

and upgrading of Jerseys to Holsteins in Australia. It is also possible that Holsteins share common ancestors with Fleckvieh (not represented in the pedigree), due to recent introgression of Red Holstein alleles into the Fleckvieh breed (Kaupe et al., 2004). Although Figure 1 shows that the breeds are genetically distinct, having common ancestors in more distant generations could increase the predictive ability of having additional breeds in a reference population in genomic selection.

In most cases, the accuracies of GEBV estimated using BayesA were superior to the accuracies of GEBV estimated using GBLUP (Figure 2). However, traitby-method-by-reference population interactions were observed, so that, in some instances, one method was better than the other. For example, fat yield was predicted more accurately using BayesA than GBLUP.
The reason for this result is likely because fat yield is known to be partly controlled by several known QTL of large effect (Grisart et al., 2002; Spelman et al., 2002) and BayesA is better able to model these effects than is GBLUP.

The results also confirmed the findings of Hayes et al. (2009) that BayesA is superior to GBLUP in achieving high accuracies of GEBV prediction across breeds. However, generally, the addition of other breeds to the reference population had only a small effect on the prediction of GEBV. For both BayesA and GBLUP, little difference existed between within-breed predictions of GEBV when the reference population included all 3 populations. This observation was consistent across traits. However, using 2 breeds in the reference to predict a third breed almost always gave higher accuracies 


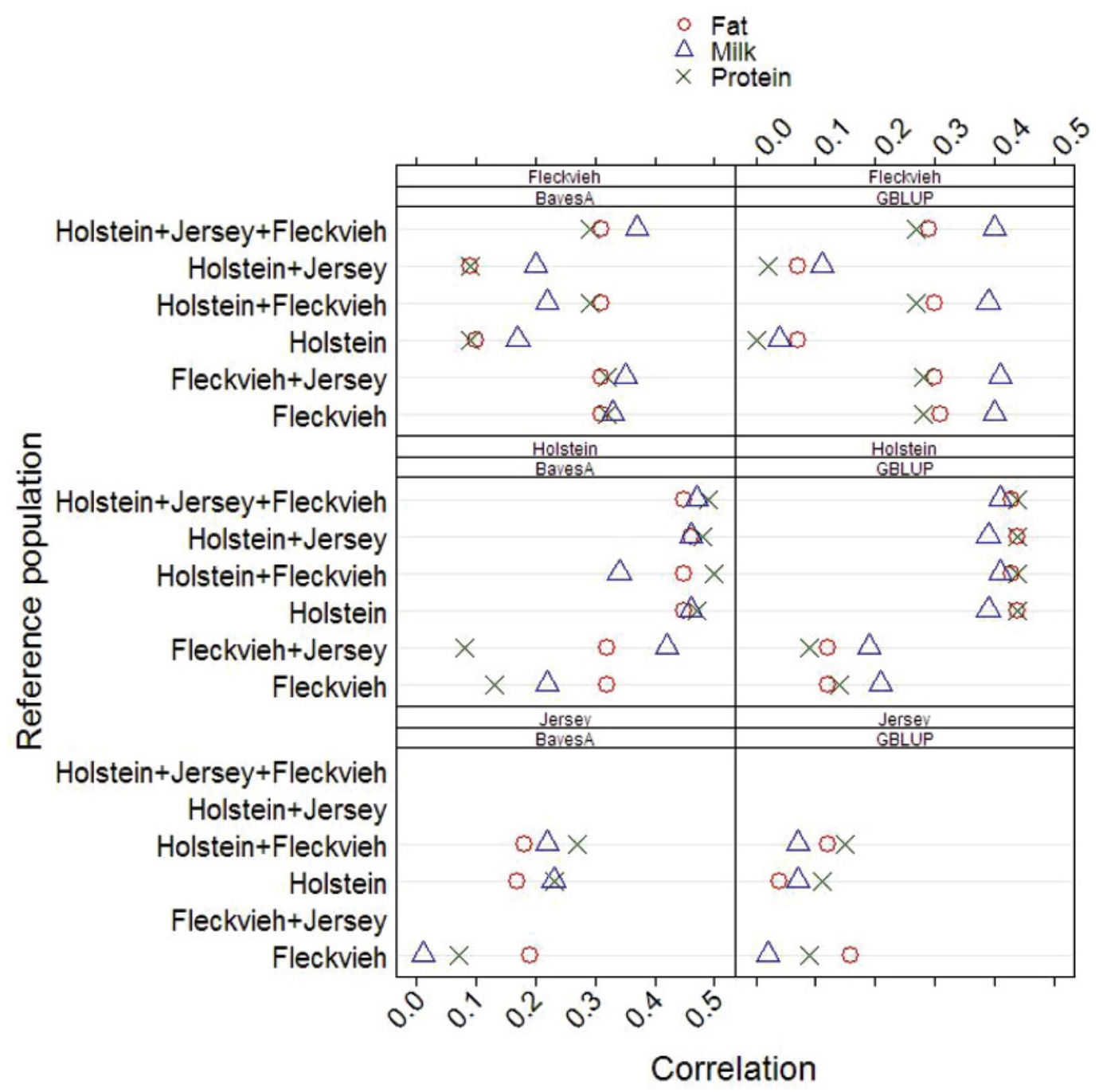

Figure 2. Accuracies of genomic EBV (GEBV) for Fleckvieh, Holstein, and Jersey validation populations estimated using a Bayesian method (BayesA) and BLUP with a multi-breed genomic relationship matrix (GBLUP) when the reference populations consisted of Fleckvieh, Holstein, and Jerseys, separately and combined. Color version available in the online PDF.

than using just 1 breed (Figure 2). For example, when Fleckvieh alone was used to predict milk GEBV (using BayesA; Figure 2) for Holsteins, the accuracy was 0.22 , whereas a Fleckvieh plus Jersey reference increased the accuracy of Holstein milk GEBV to 0.42 .

Both Ibánẽz-Escriche et al. (2009) and De Roos et al. (2009) concluded that across-population evaluations were preferable to within-population evaluations when the populations were closely related, marker density was high, or the number of animals with phenotypic records was small. The more genetically divergent populations are, the greater the marker density must be to preserve marker-QTL phase across populations (de Roos et al., 2009). So, for the marker density in the present study, the populations were likely to be too divergent to detect enough marker-QTL associations persisting across breed to explain a large proportion of the genetic variance. The interval between adjacent markers on the Illumina BovineSNP50 BeadChip is around 67 kbp (Matukumalli et al., 2009). At this interval, the LD measure $\left(\mathrm{r}^{2}\right)$ is $0.20,0.18$, and 0.15 in Holsteins, Jerseys, and Fleckvieh, respectively (Figure 3). The overall pattern of LD levels for marker distances from 10 to $1,000 \mathrm{kbp}$ indicates a much lower persistence of LD for Fleckvieh than for the 2 other breeds.

Hayes et al. (2009) suggested that the reason why using more than one breed in a reference population could exceed a single-breed reference population is that for an SNP to have an effect in a multi-breed reference population it must be in high LD with QTL in all breeds in the reference population. This may be more obvious when investigating specific genomic regions 


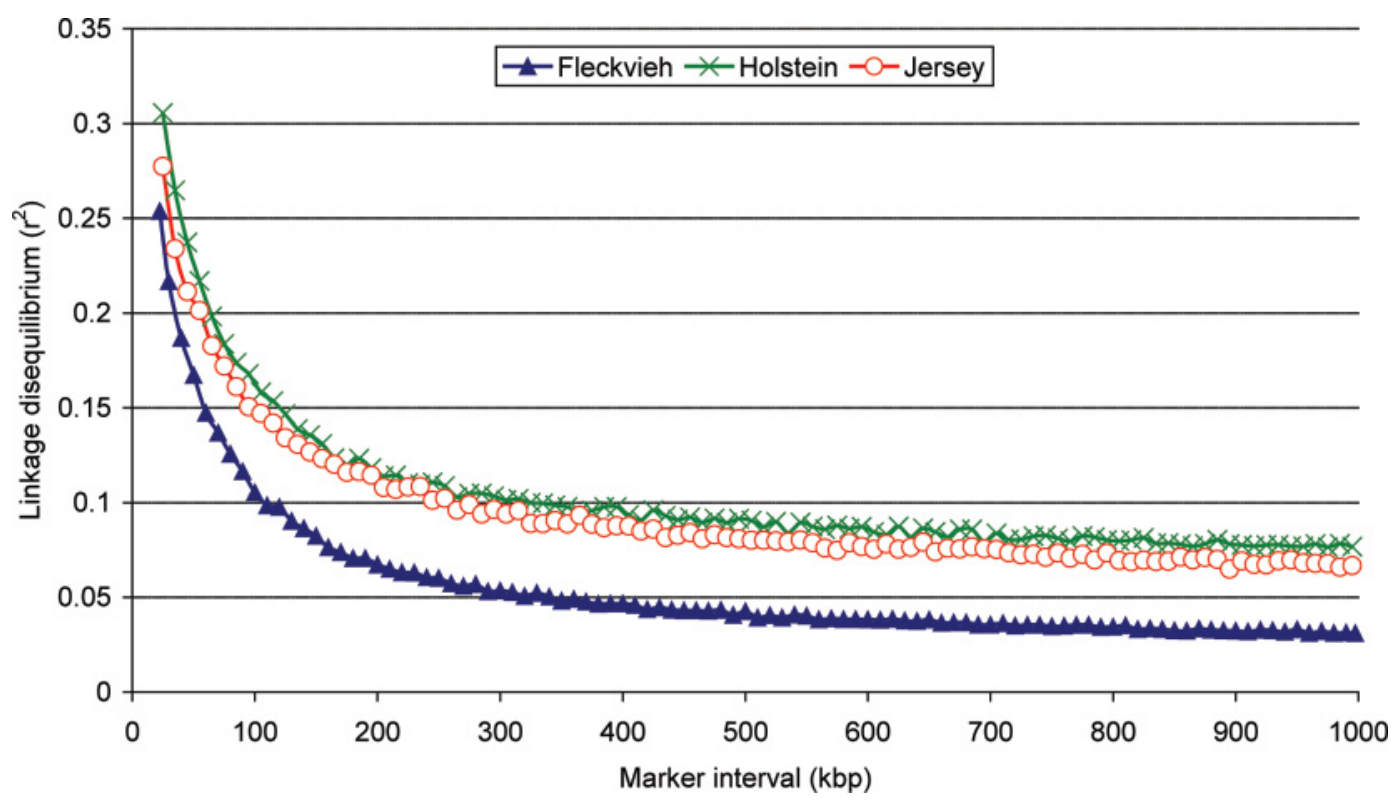

Figure 3. Linkage disequilibrium, measured as $\mathrm{r}^{2}$ between syntenic markers in Fleckvieh, Holstein, and Jersey populations. Color version available in the online PDF.

known to have an effect on the traits studied, rather than detecting an increase in accuracy. To see if this was the case in the data set used here, SNP effects for fat yield estimated using BayesA were selected from a region on chromosome 14 close to the DGAT1 mutation. The DGAT1 mutation has a known major effect on the fat concentration in milk in all 3 breeds included in this study (Grisart et al., 2002; Spelman et al., 2002; Kaupe et al., 2004). In the single-breed reference populations (Fleckvieh and Holstein), the effect of the DGAT1 mutation is captured by $2 \mathrm{SNP}, 1$ that is in the close vicinity of DGAT1 and the other that is around $200 \mathrm{kbp}$ away (Figure 4). In the Holstein-Fleckvieh and Holstein-Fleckvieh-Jersey reference populations, other SNP are filtered, so that only 1 SNP is in high LD with DGAT1 (Figure 4). This demonstrates that the BayesA method will predict effects associated with specific QTL more precisely in a multi-breed population compared with a single-breed population.

Using multiple breeds to predict GEBV is an attractive way to increase the size of population used to calculate prediction equations in genomic selection. However, in the Holstein, Jersey, and Fleckvieh dairy cattle populations used here, the accuracy of GEBV from multi-breed reference populations was not greater than that achieved from single-breed reference populations. This is because these specific populations are too diverged for the SNP density used here to capture enough marker-QTL associations, which persist across breeds due to LD. Another reason could be genotype by environment interactions and differences in methods of genetic evaluation, meaning that the addition of other breeds did not improve genomic predictions. However when the goal was to predict genomic breeding values for a breed with no individuals in the reference popu-

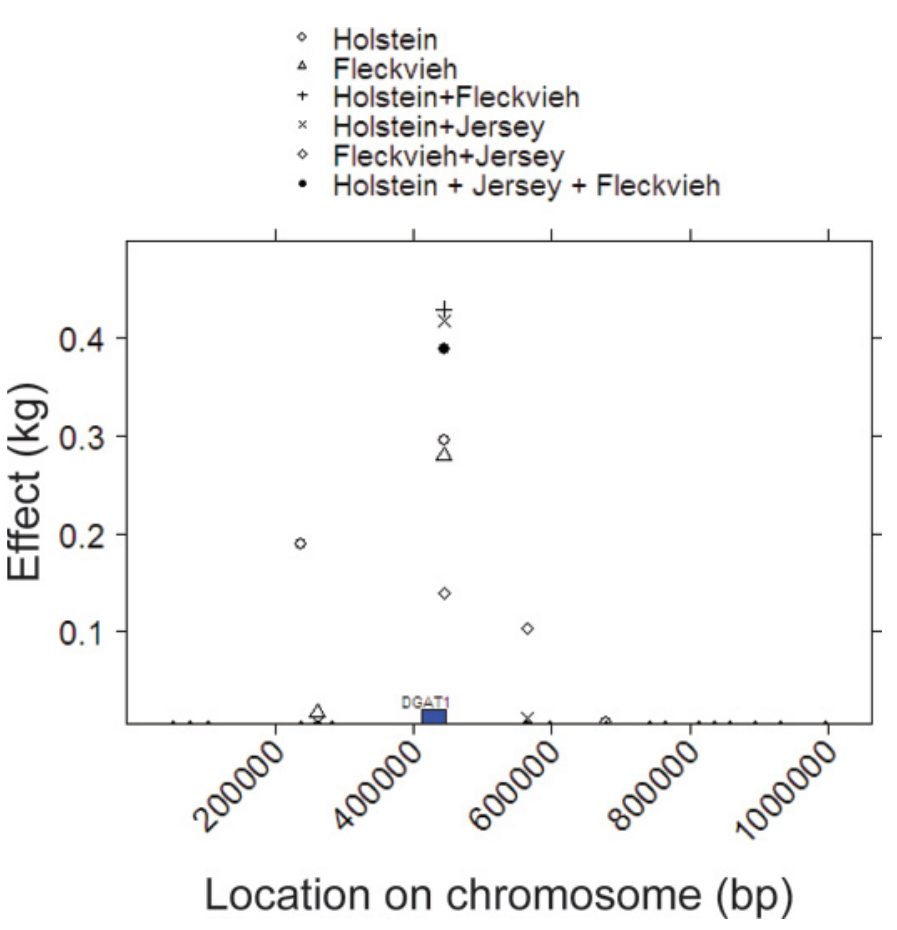

Figure 4. Size of SNP effects (ignoring sign) for fat yield estimated using a Bayesian method (BayesA) in the reference populations (Holstein, Fleckvieh, Holstein + Fleckvieh, Holstein + Jersey, Fleckvieh + Jersey, and Holstein + Jersey + Fleckvieh) close to the $D G A T 1$ gene on chromosome 14. Color version available in the online PDF. 
lation, using 2 (different) breeds in the reference was generally better than using a single breed.

\section{ACKNOWLEDGMENTS}

The Australian authors thank the Australian Dairy Herd Improvement Scheme (ADHIS; Melbourne, Victoria, Australia) for providing the phenotype data used in this study. We are also grateful to Curt van Tassell and Tad Sonstegard from the USDA (Beltsville, MD) for providing genotypes of Australian bulls, under a collaborative agreement between the USDA and the Department of Primary Industries Victoria. The Austrian and German authors thank the Federation of Austrian Fleckvieh Cattle Breeders (AGÖF; Zwettl, Austria) for providing the genotypes of Austrian and German bulls.

\section{REFERENCES}

de Roos, A. P. W., B. J. Hayes, R. Spelman, and M. E. Goddard. 2009. Reliability of genomic breeding values across multiple populations. Genetics 183:1545-1553.

Gilmour, A. R., B. J. Gogel, B. R. Cullis, S. J. Welham, and R. Thompson. 2006. ASReml User Guide Release 2.0. VSN International Ltd., Hemel Hempstead, UK.

Grisart, B., W. Coppieters, F. Farnir, L. Karim, C. Ford, P. Berzi, N. Cambisano, M. Mni, S. Reid, P. Simon, R. Spelman, M. Georges, and R. Snell. 2002. Positional candidate cloning of a QTL in dairy cattle: Identification of a missense mutation in the bovine DGAT1 gene with major effect on milk yield and composition. Genome Res. 12:222-231.

Hayes, B. J., P. J. Bowman, A. C. Chamberlain, K. Verbyla, and M. E. Goddard. 2009. Accuracy of genomic breeding values in multibreed dairy cattle populations. Genet. Sel. Evol. 41:51.

Hayes, B. J., and M. E. Goddard. 2008. Technical note: Prediction of breeding values using marker-derived relationship matrices. J. Anim. Sci. 86:2089-2092.

Hill, W. G., and A. Robertson. 1968. Linkage disequilibrium in finite populations. Theor. Appl. Genet. 38:226-231.

Ibánẽz-Escriche, N., R. L. Fernando, A. Toosi, and J. C. M. Dekkers. 2009. Genomic selection of purebreds for crossbred performance. Genet. Sel. Evol. 41:12.

Kaupe, B., A. Winter, R. Fries, and G. Erhardt. 2004. DGAT1 polymorphism in Bos indicus and Bos taurus cattle breeds. J. Dairy Res. 71:182-187.

Matukumalli, L. K., C. T. Lawley, R. D. Schnabel, J. F. Taylor, M. F. Allan, M. P. Heaton, J. O'Connell, S. S. Moore, T. P. L. Smith, T. S. Sonstegard, and C. P. Van Tassell. 2009. Development and characterization of a high density SNP genotyping assay for cattle. PLoS One 4:e5350.

McVean, G. 2009. A genealogical interpretation of principal components analysis. PLoS Genet. 5:e1000686. doi:10.1371/journal. pgen. 1000686

Meuwissen, T. H. E., B. J. Hayes, and M. E. Goddard. 2001. Prediction of total genetic value using genome-wide dense marker maps Genetics 157:1819-1829.

Spelman, R. J., C. A. Ford, P. McElhinney, G. C. Gregory, and R. G. Snell. 2002. Characterization of the DGAT1 gene in New Zealand dairy population. J. Dairy Sci. 85:3514-3517.

VanRaden, P. M., C. P. Van Tassell, G. R. Wiggans, T. S. Sonstegard, R. D. Schnabel, J. F. Taylor, and F. S. Schenkel. 2009. Invited review: Reliability of genomic predictions for North American Holstein bulls. J. Dairy Sci. 92:16-24. 